\title{
Die großen wissenschaftlichen Leistungen von Stefan Vogel (1925-2015) Teil 5a. Öl statt Nektar - die Ölblumen (Allgemeine Aspekte)
}

\author{
Anton Weber, Günter Gerlach \& Stefan Dötterl
}

\begin{abstract}
After the discovery of perfume flowers, STEFAN Vogel added another new floral type to botanical science: the oil flowers. In these flowers, fatty oil is presented as a floral reward. The oil is produced in the so-called elaiophores and collected by specialised bees (members of several groups of Apidae-Apinae and two genera of Melittidae-Melittinae) possessing highly elaborate collecting structures on the legs or the abdomen. The oil is used, mixed with pollen, as a food for the larvae or, in pure condition, for cell lining. In more than 20 (including three book-like) publications Vogel proved the existence of oil flowers in 10 plant families of predominantly tropical and subtropical distribution (another one was added by Simpson \& NefF 1981) and described the details of the flower and their bee pollinators. In the present article, general aspects of oil flowers (history of discovery and exploration, distribution in the angiosperms, basic types of elaiophores, utilisation, oil chemistry, oil-collecting bees) are treated. A selection of oil-flowers will be described and illustrated in the next issue of this journal.
\end{abstract}

\section{Zusammenfassung}

Nach der Entdeckung der Parfümblumen hat Stefan Vogel einen weiteren neuen Blumentypus erkannt und erforscht: die Ölblumen. Hier wird von den Blüten fettes Öl als Sammelgut angeboten, das in den sogenannten Elaiophoren gebildet wird. Das Öl wird von spezialisierten Bienen (Vertreter mehrerer Gruppen der Apidae-Apinae und zwei Gattungen der Melittidae-Melittinae) mit hochdifferenzierten Sammelstrukturen an den Beinen oder am Hinterleib gesammelt, und, mit Pollen vermischt, als Futter für die Brut verwendet. In reiner Form wird es auch zum Imprägnieren der Brutzellen genutzt. In über 20, teils sehr umfangreichen Publikationen hat Vogel Ölblumen in 10 Familien meist tropisch- und subtropischer Verbreitung nachgewiesen, beschrieben und den Bestäubungsmodus aufgeklärt (eine weitere Familie bzw. Gattung wurde von Simpson \& Neff 1981 hinzugefügt). In diesem Beitrag werden allgemeine Aspekte der Ölblumen (Entdeckungs- und Erforschungsgeschichte, Vorkommen bei den Angiospermen, Grundtypen von Elaiophoren, Nutzung des Öls, Öl-Chemie, Überblick über ölsammelnde Bienen), dargelegt. In der nächsten Ausgabe dieser Zeitschrift wird eine Auswahl von Ölblumen näher beschrieben und illustriert.

\section{Einleitung}

Mit den Parfümblumen hatte Stefan Vogel (1966) einen neuen Blütentypus entdeckt, bei dem nicht Pollen oder Nektar als Sammelgut fungiert, sondern ätherisches Öl, also flüchtige Duftstoffe, die in Form von kleinen Tröpfchen von den sogenannten Osmophoren abgesondert und von Prachtbienenmännchen aktiv gesammelt werden (vgl. Weber \& Gerlach 2018). Dass auch fettes Öl an Stelle von Pollen oder Nektar als Sammelgut dienen kann, ist eine weitere großartige Entdeckung von Stefan Vogel. Diesem biologischen Phänomen ist er mit einer Konsequenz und Beharrlichkeit nachgegangen, die ihresgleichen sucht. Er hat nicht nur die botanischen Aspekte in allen möglichen Details aufgeklärt, sondern hat sich auch unsterbliche Verdienste auf zoologischer Seite erworben. Im Laufe der Zeit hat er über Ölblumen und ölsammelnde Bienen an die 25 Artikel veröffentlicht, darunter drei in buchartig-monographischer Form, bekannt als die sogenannte Ölblumen-Trilogie. Und dabei hat alles so harmlos und unspektakulär begonnen ...

\section{Ausgangspunkt: Nektar ohne Zucker}

Die Erforschung der Ölblumen geht auf eine scheinbar nebensächliche Beobachtung zurück, die zudem Vogel gar nicht selbst gemacht hat. Anfang der 1960er Jahre machte Dimitri Harte, Vogels Kollege am Institut für Spezielle Botanik der Universität Mainz, die merkwürdige Beobachtung, dass der Nektar von südamerikanischen Angelonia-Arten, die im Botanischen Garten kultiviert wurden, keinen Zucker enthält (siehe Troll 1963, 1965, Vogel 1974, 1989). Hartl wandte sich deshalb an Stefan Vogel, ob er dazu etwas wüsste. Das war nicht der Fall. Jeder andere Botaniker hätte wahrscheinlich die Sache auf sich beruhen lassen und HarTLs Beobachtung als Kuriosität am Rande abgetan. Nicht so Vogel. Er begann den Verwandtschaftskreis von Angelonia genauer $\mathrm{zu}$ untersuchen und führte verschiedene einfache physikalische und chemische Experimente durch. Tatsächlich, so stellte er fest, bildeten sich Schlieren (sogenannte Myelin-Figuren), wenn man dem Nektar unter dem Mikroskop einen Tropfen 
Wasser zufügte. Damit war ein erster Nachweis erbracht, dass der Nektar aus fettem Öl bestehen müsse.

Schlagartig wurde Vogel klar, dass er hier einer neue Klasse von floralen Belohnungsmitteln auf der Spur war. Damit war der Weg für die weitere Beschäftigung mit derartigen Blüten vorgezeichnet. Auf seiner Kolumbien-Reise 1964 hatte er Gelegenheit, weitere ölsezernierende Blüten (insbesondere Calceolaria-Arten) zu studieren und konnte die Bestäubung von Angelonia durch Bienen der Gattung Centris nachweisen (siehe Troll 1965). Auch bei zwei weiteren Familien, den Malpighiaceen und einer Gattung der Orchideen (Oncidium), konnte er feststellen, dass von den Blüten fettes Öl anstelle von herkömmlichem, zuckerhaltigem Nektar als flüssiges Attraktans dargeboten wird.

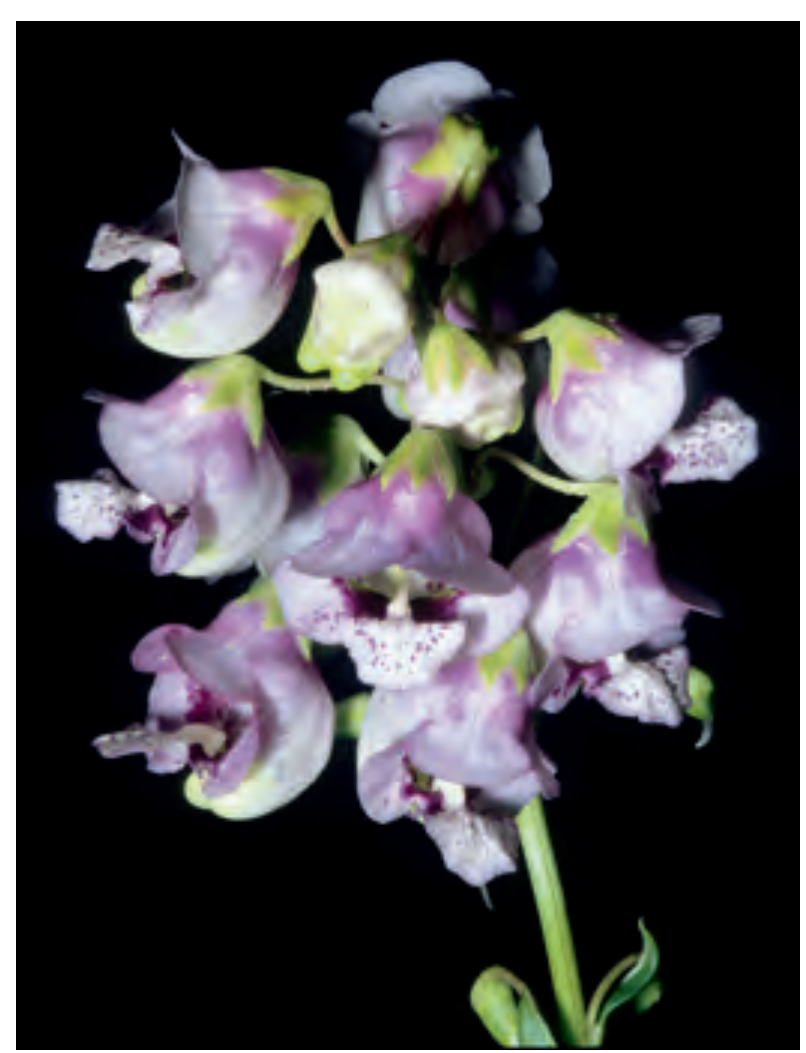

Abb. 1: Blütenstand von Angelonia integerrima (Plantaginaceae). Angelonia-Arten gaben den Anstoß zur Entdeckung und Erforschung der Ölblumen durch Stefan Vogel. An ihnen gelang Vogel auch erstmals der Nachweis, dass speziell angepasste Bienen (im gegenständlichen Fall Arten der Gattung Centris) das florale Öl sammeln und dabei die Bestäubung durchführen. (Argentinien, Prov. Entre Rios, Parque Nacional El Palmar, cult. BG-München-Nymphenburg 6/2003). (Foto: G. Gerlach)
Im Jahr 1969 trat Stefan Vogel mit diesen Ergebnissen zum ersten Mal vor die wissenschaftliche Öffentlichkeit. Im Rahmen des 11. Internationalen Botanischen Kongresses in Seattle (USA, Aug./Sept. 1969) hielt er ein Referat mit dem prägnanten Titel „Flowers offering fatty oil instead of nectar".

Das war der Anfang. Was noch folgen sollte, hätte sich Vogel zu diesem Zeitpunkt wohl selbst nicht träumen lassen, nämlich die Entdeckung von Ölblumen in mehr als 10 Pflanzenfamilien und etwa 1800 Arten, quer durch das System der Blütenpflanzen und quer über die großen Kontinente hinweg.

\section{Vorkommen und Erforschungsgeschichte der Ölblumen}

Wie bereits erwähnt, nahm die Entdeckung der Ölblumen ihren Ausgang von Beobachtungen bei Arten der Gattung Angelonia. Damals wurde die Gattung zu den Braunwurzgewächsen (Scrophulariaceae) gestellt und so lag es nahe, diese Familie näher unter die Lupe zu nehmen. Tatsächlich fand Stefan Vogel eine Reihe von weiteren Gattungen, namentlich Calceolaria, Diascia, Bowkeria und Anastrabe, deren Blüten ebenfalls fettes Öl produzieren. Heute werden diese Gattungen in verschiedene Familien gestellt: Angelonia zu den Plantaginaceae, Calceolaria zu den Calceolariaceae, Diascia zu den Scrophulariaceae, Bowkeria und Anastrabe zu den Stilbaceae. Die Familien stehen sich allerdings nahe und gehören alle der Ordnung Lamiales an. Aber auch in anderen Verwandtschaftskreisen, welche nicht mit den Lamiales verwandtschaftlich verbunden sind, wurde Vogel fündig: bei den Dikotyledonen in den Familien der Malpighiaceae (fast alle neotropischen Gattungen!) und Krameriaceae (mit der einzigen Gattung Krameria), sowie bei den Monokotyledonen in den Schwertliliengewächen (Iridaceae: Alophia, Cypella, Sisyrichnium und "Sphenostigma“ = Ennealophus) und - wie könnte es anders sein den Orchideen (Oncidium, Sigmatostalix u. a.).

Nachdem Vogel anlässlich eines Kongresses und in zwei Kurzpublikationen die Existenz der Ölblumen sozusagen offiziell vorgestellt hatte 
(Vogel 1969, 1971, 1973), publizierte er 1974 die groß angelegte Darstellung „Ölblumen und ölsammelnde Bienen“, eine buchförmige Publikation mit 267 Seiten, zahlreichen exzellenten Zeichnungen und Fototafeln.

Doch kurz vorher hatte Vogel schon eine neue Entdeckung gemacht: Ölblumen gab es nicht nur in den Tropen und Subtropen, sondern auch in der Holarktis, Mitteleuropa mit eingeschlossen! Jeder botanisch Interessierte kennt die Familie und die Gattung: die Primelgewäche (Primulaceae) bzw. den Gilbweiderich (Lysimachia). Doch blieb es der scharfen Beobachtungsgabe von Stefan Vogel vorbehalten, dass viele (wenn auch nicht alle) Arten von Lysimachia fettes Öl als florales Attraktans produzieren. Als Bestäuber konnte VogeL die Arten der hochspezialisierten Schenkelbienen (Macropis) ausmachen. Wieder stellte Vogel die neue Entdeckung in einem Kongressreferat und in einer Kurzpublikation der wissenschaftlichen Öffentlichkeit vor (Vogel 1976a, b), um dann zehn Jahre später eine großangelegte Darstellung folgen zu lassen, nämlich die zweite Folge der Ölblumen: "Lysimachia und Macropis" (Vogel 1986, 161 Seiten). Zum ersten Mal verwendete Vogel hier rasterelektronenmikroskopische Aufnahmen zur Dokumentation der Sammelstrukturen der Bienen.

Um die gleiche Zeit (die ersten Hinweise hatte Vogel schon 1974 gesammelt) zeichnete sich ein neues Forschungsfeld hinsichtlich Ölblumen ab. Die Blüten der altweltlich-tropischen Gattungen Momordica und Thladiantha (Kürbisgewächse, $\mathrm{Cu}$ curbitaceae) und ihre Bestäubung durch Bienen der Gattung Ctenoplectra. Zunächst stellte VogeL den eigentümlichen Sammelvorgang (Ölschwänzeln) in den Mittelpunkt einer Kurzpublikation (Vogel 1981), bevor er 1990 die dritte Folge der Ölblumen publizierte mit dem Titel „Momordica, Thladiantha und die Ctenoplectridae" (186 Seiten).

$\mathrm{Zu}$ den Untersuchungen Vogels ist eigentlich nur eine Familie hinzugekommen, in der sich ölblütige Vertreter finden, nämlich die Nachtschattengewächse (Solanaceae) mit Nierembergia
(Simpson \& NefF 1981). Aber auch hier gibt es einen Bezug zu Vogel: Die Bestäubung von Nierembergia wurde im Detail von VogeLs argentinischem Schüler Andrea Cocucci untersucht (Cocucci 1991).

Der Vollständigkeit halber seien noch drei weitere Familien genannt, bei denen in den Blüten Öl gefunden wurde, das aber hier nicht als Sammelgut dient, nämlich die Gesneriaceae, Melastomataceae und Myrtaceae. Bei Drymonia-Arten (Gesneriaceae) dient das (spärliche) Öl anscheinend dem Anheften des Pollens an den Bestäuber (Steiner 1985, Steiner \& Whitehead 1991), ähnliches gilt vielleicht auch für Emarhendia (Vogel in Kiew et al. 1998). Für die Melastomataceen geben Buchmann \& Buchmann (1981) an, bei Mouriri myrtilloides florales Öl gefunden zu haben und schließen daraus, dass die ganze Tribus Memecyleae (350 - 400 Arten) ölblütig sei. Diese Angaben sind jedoch stark anzuzweifeln (vgl. Розуовом \& Machado 2017), bei Renner \& Schaefer (2010) wird die Familie gar nicht erwähnt. Bei den Myrtaceen haben Houston et al. (1993) in der Gattung Verticordia eine ölartige Substanz in den Antheren gefunden, in der die Pollenkörner eingebettet sind. Es handelt sich hier um eine Art ölig-zähflüssigen Pollenkitt.

In nachfolgender Tabelle 1 sind die Ordnungen, Familien und wichtigsten Gattungen mit Ölblumen zusammengestellt. Nach den bisher vorliegenden Daten lassen sich folgende Feststellungen treffen:

(1) Ölblumen im klassischen Sinn kommen in 9 Ordnungen, 11 Familien und schätzungsweise 75-80 Gattungen der Angiospermen vor.

(2) Meist ist es nur eine Familie pro Ordnung, in der sich Ölblumen entwickelt haben, eine Ausnahme bilden nur die Lamiales mit 4 Familien.

(3) In den meisten Familien haben sich selbst innerhalb der Familie Gattungen mit Ölblumen mehrfach parallel entwickelt. 


\begin{tabular}{|c|c|c|}
\hline Ordnung & Familie & $\begin{array}{l}\text { Wichtige Gattungen } \\
\text { In Klammer: (z.T. ungefähre) Zahl der Arten/Zahl der } \\
\text { ölblütigen Arten }\end{array}$ \\
\hline Liliales & Iridaceae & $\begin{array}{l}\text { Alophia (5/5), Cypella (20/20), Ennealophus (5/5), Gelasine (4/4), } \\
\text { Sisyrichnium (ca. 100/35), Tigridia (35/1), Trimezia (20/20) }\end{array}$ \\
\hline Orchidales & Orchidaceae & $\begin{array}{l}\text { Paläotropisch: Ceratandra (5/6), Corycium (14/14), } \\
\text { Disperis (75/75), Huttonaea (5/5), Pterygodium (18/18) } \\
\text { Neotropisch: Maxillaria (420/20), Oncidium (680/50), Ornitho- } \\
\text { cephalus (50/50), Sigmatostalix (35/35), Zygostates (24/24) }\end{array}$ \\
\hline Zygophyllales & Krameriaceae & Krameria $(17 / 17)$ \\
\hline Malpighiales & Malpighiaceae & $\begin{array}{l}\text { Fast alle neotropischen Gattungen, darunter die folgenden be- } \\
\text { kannten und/oder artenreiche Gattungen: } \\
\text { Banisteriopsis }(92 / 92) \text {, Bunchosia }(60 / 60) \text {, Byrsonima (150/150), } \\
\text { Heteropteris (120/120), Hiraea (45/45), Malpighia (30/30), Ma- } \\
\text { scagnia (55/55), Stigmaphyllon (100/100), Tetrapterys (90/90) }\end{array}$ \\
\hline Cucurbitales & Cucurbitaceae & Indofevillea (2/1), Momordica (45/45), Thladiantha (23/23) \\
\hline Ericales & Primulaceae & Lysimachia $(150 / 75)$ \\
\hline Solanales & Solanaceae & Nierembergia (23/5), (21/ „most spp.“- - TATE et al. 2009) \\
\hline \multirow[t]{4}{*}{ Lamiales } & Calceolariaceae & Calceolaria $(260 / 210)$ \\
\hline & Plantaginaceae & $\begin{array}{l}\text { Angelonia }(25 / 25), \text { Basistemon (8/8), Monopera (2/2), } \\
\text { Monttea (3/3) }\end{array}$ \\
\hline & Scrophulariaceae & Alonsoa (18/2), Colpias (1/1), Diascia (48/38), Hemimeris (4/4) \\
\hline & Stilbaceae & Anastrabe (1/1), Bowkeria (5/5), Ixianthes (1/1) \\
\hline
\end{tabular}

Tab. 1: Die Ordnungen und Familien der Angiospermen mit Ölblumen. Artenzahlen (Gesamtzahl in der Gattung/Arten mit Ölblumen). Daten aus Vogel (1974, 1984, 1990), Goldblatt et al. (1998), Steiner \& Whitehead (1990, 1991), Whitehead \& Steiner (1992), Rasmussen \& Olesen (2000), Renner \& Schaefer (2010) und Schaefer et al. (2012).

(4) Insgesamt beträgt die Zahl der Arten mit Ölblumen etwa 1800, davon entfallen mehr als 800 Arten auf die Malpighiaceen und über 200 auf Calceolaria.

(5) Was die geografische Verbreitung betrifft, kommen mehr als 4/5 der Ölblumen-Arten in den Neotropen vor.

\section{Elaiophoren, die Ölfabriken in den Blüten} Eine der ersten Fragen, die sich Vogel naturgemäß stellte, war, wo und wie das Öl in der Blüte produziert wird. Es sind dies lokale Drüsenfelder, die sich durch übermäßige Produktion und Sekretion fettartiger Substanzen (Lipide) auszeichnen. VoGel (1969) prägte für sie den Begriff Elaiophoren („Ölträger"). Sie sind durchwegs epidermaler Herkunft. Die Blüten sondern in der Regel entweder Nektar oder Öl ab, nicht aber beides zugleich (Ausnahme: Cucurbitaceen).

Hinsichtlich der anatomischen Struktur konnte Vogel (1974) zwei verschiedene Typen feststellen: (1) die Trichom-Elaiophoren und (2) die Epithel-Elaiophoren. 
Die Trichom- oder Haar-Elaiophoren der Dikotyledonen bestehen aus Drüsenhaaren, die in Stiel und Köpfchen gegliedert sind. Sie können innerhalb gewisser Bezirke locker und diffus verteilt stehen, häufiger stehen sie aber sehr eng (d. h. aus jeder Epidermiszelle geht ein Drüsenhaar hervor) und bilden mehr oder weniger scharf begrenzte Felder. Das Öl wird in den mehrzelligen, länglichen oder kugeligen Köpfchen gebildet. Es sammelt sich zunächst unter der Cuticula an. Diese platzt ohne Einwirkung der Bestäuber auf. Das Öl findet sich dann in Form von Tröpfchen an den Köpfchen wieder oder lagert sich zwischen den Härchen ab. Es wird nie in speziellen Safthaltern gelagert, wie das bei vielen Nektarblumen der Fall ist. Trichom-Elaiphoren können an verschiedenen Stellen der Blüte sitzen: auf dem Kelch, der Blumenkrone, hier oft in sack-, schuh- oder spornförmigen Ausbuchtungen, oder auf der Krone und den Filamenten der Staubblätter (Lysimachia). Bei den Pantoffelblumen (Calceolaria-Arten) sitzen die Elaiophoren im Inneren des Pantoffels. Die ölsammelnden Bienen müssen komplizierte Bewegungen vollführen, um an das Öl zu gelangen. Trichom-Elaiophoren sind typisch für die Lamiales (Calceolariaceae, Plantaginaceae, Scrophulariaceae und Stilbaceae), die Primulaceae (Lysimachia) und Cucurbitaceae (Momordica, Thladiantha). In allen Fällen ist hier der Stiel und/ oder das Köpfchen der Ölhaare mehrzellig. Bei den Monokotyledonen sind Trichom-Elaiophoren für die Iridaceen typisch, vereinzelt auch bei den Orchideen zu finden (z. B. Zygostates). Hier sind die Drüsenhaare einzellig, bestehen also nur aus schlauchförmig ausgewachsenen Epidermiszellen. Manchmal sind sie im apikalen Teil etwas kopfig erweitert, bilden aber nie echte, mehrzellige Köpfchen.

Die Epithel-Elaiophoren bestehen nicht aus Haaren, sondern bilden geschlossene Decken aus meist senkrecht gestellten, prismatischen Säulenzellen. Die Zwischenwände der Säulenzellen können sich voneinander lösen oder sich mehr oder weniger ganz auflösen, sodass ein Polster aus Pseudo-Haaren entsteht. Die abschließende Cuticula bleibt erhalten, sie wird durch das sezernierte Öl abgehoben. Dieses tritt durch anscheinend vor-

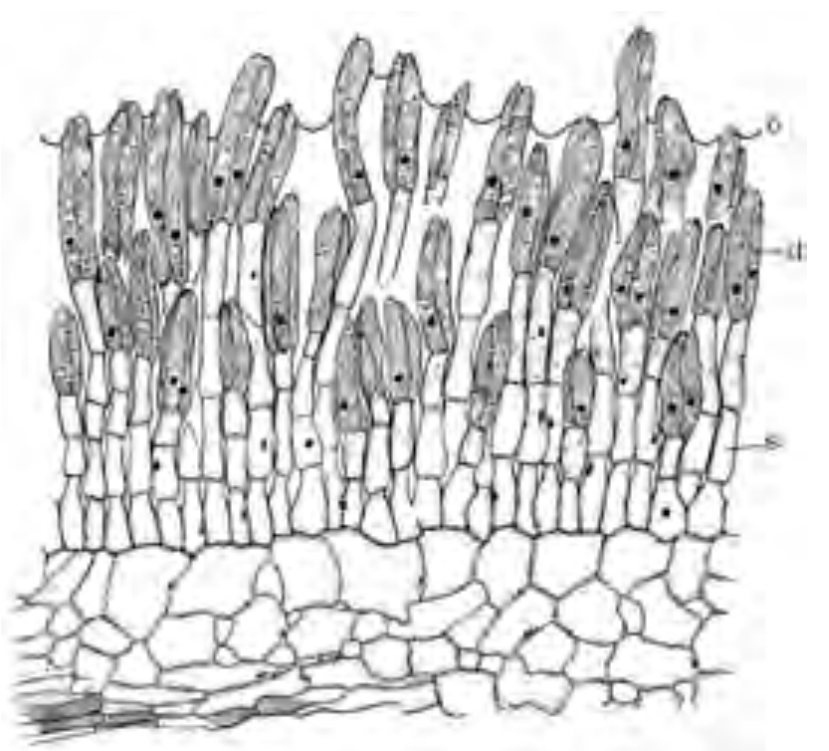

Abb. 2: Schnitt durch den Trichom-Elaiophor von Calceolaria pavonii (Calceolariaceae). Sowohl der Stiel (s) wie auch das längliche Köpfchen (d) bestehen aus mehreren Zellen: ö Ölspiegel (aus Vogel 1974, Abb. 2A).

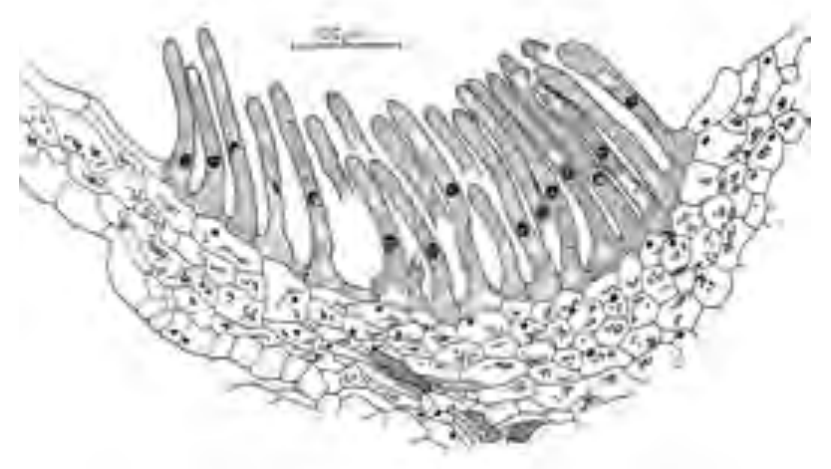

Abb. 3: Schnitt durch den Trichom-Elaiophor von Zygostates pustulata (Orchidaceae). Die ölsezernierenden Haare sind einzellig und stellen die ausgewachsenen Epidermiszellen des Blütenblattes dar (aus Vogel 1974, Abb. 23A).

geformte Dünnstellen aus. Es lagert dann auf der Cuticulardecke und wird von dort von den Bienen abgesammelt.

Epithel-Elaiophoren sind charakteristisch für die Malpighiaceen, Krameriaceen und einige Orchideen. Am auffälligsten sind sie bei den Malpighiaceen, wo sie vergleichsweise riesige Drüsenkörper an den Kelchblättern bilden. Manchmal ist das Kelchblatt soweit reduziert, dass es nur aus den Drüsenkörpern zu bestehen scheint. Die geschilderten Drüsenkörper sezernieren nur bei den neuweltlichen Arten Öl. Bei den altwelt- 


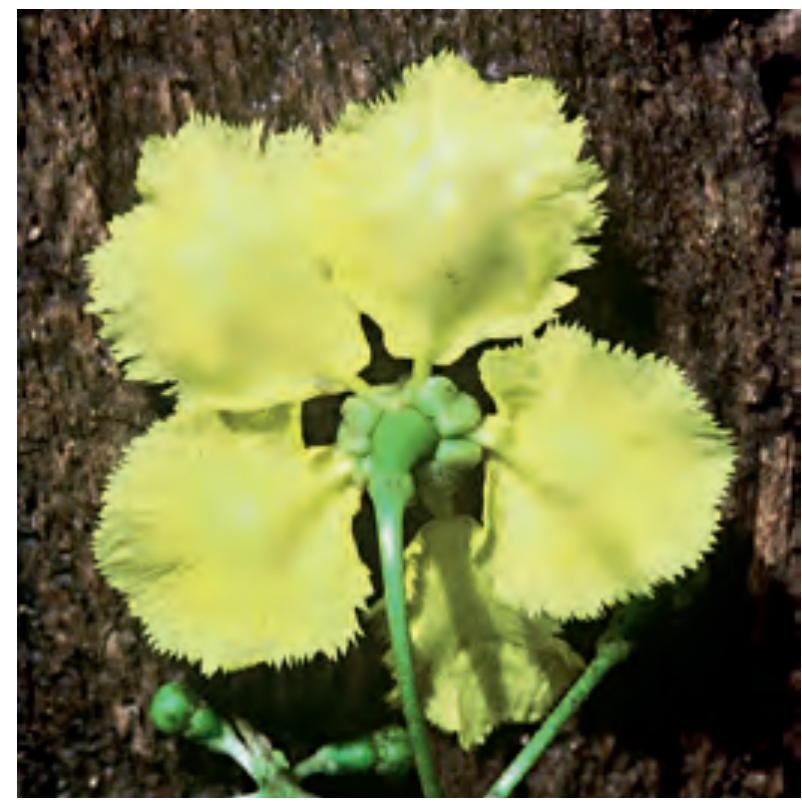

Abb. 4: Stigmatophyllon ellipticum, Blüte von unten gesehen. Zwischen den verschmälerten Kronblattbasen (Nägeln) befinden sich die meist paarigen Kelchdrüsen (Epithel-Elaiophoren). (Foto: A. WEBER)

lichen (afrikanischen, madagassischen und südost-asiatischen) Vertretern, die zusammen nur etwa $15 \%$ der Arten ausmachen, sind die anatomisch gleich gebauten Drüsen steril, sondern Nektar ab oder fehlen.

\section{Florales Öl, wofür?}

Für den Leser hat sich sicher schon längst die Frage aufgedrängt, wozu das Öl von den Bienen verwendet wird. Auf Grund der Nichtflüchtigkeit und weitgehenden Geruchlosigkeit (zumindest für die menschliche Nase) dient es sicher nicht der Fernanlockung der Bienen. Als Nahrungsmittel käme es schon eher in Betracht, zumal Öl wesentlich energiereicher ist als Nektar, der bekanntlich als Energielieferant und „Sprit“ für die blütenbesuchenden Insekten dient. Wäre es so, müssten eigentlich Männchen und Weibchen gleichermaßen das Öl konsumieren. Das ist aber nicht der Fall: das Öl wird nur von den Weibchen gesammelt. Es dient, wie Vogel zeigen konnte, in erster Linie der Verproviantierung der Brut. Das Öl wird mit Pollen vermischt und in die Nester eingetragen. Dort wird es in den Brutzellen als Larvenfutter als eine Art „Ölkuchen“ bereitgestellt. Ähnlich ist es ja auch mit dem Nektar: die Bienen-Weib-

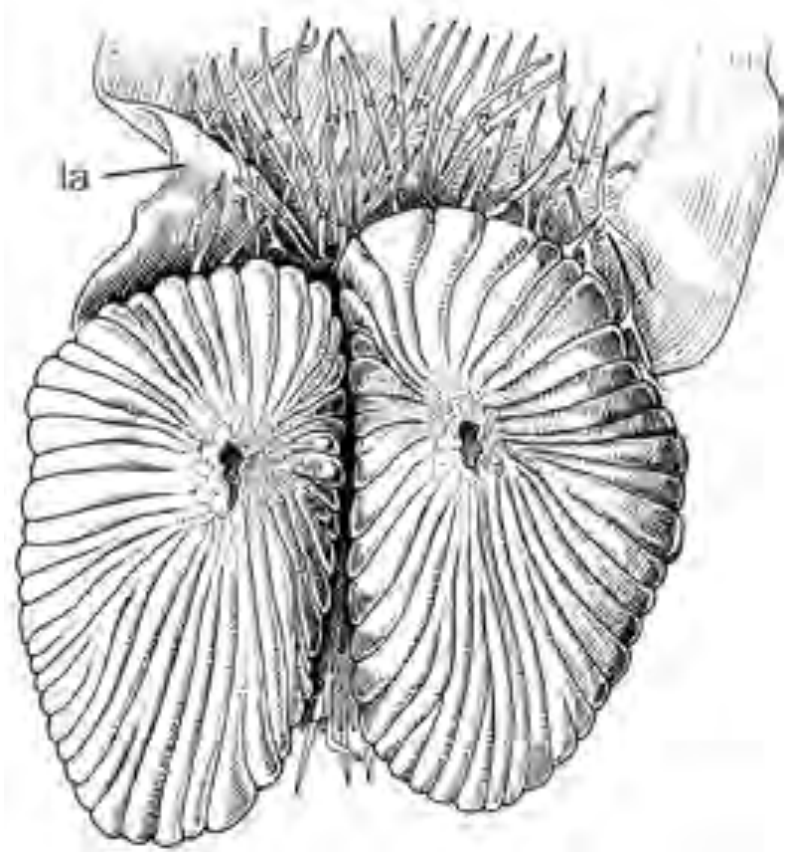

Abb. 5: Kelchblatt von Stigmaphyllon littorale (Malpighiaceae) mit paarig angeordneten Elaiophoren; la Spreite (Lamina) des Kelchblattes (aus Vogel 1974, Abb. 25A).

chen verwenden nur einen Teil des aufgesaugten Nektars zur Eigenversorgung, ein anderer Teil wird mit Pollen vermischt und als Larvenproviant verwendet.

Eine andere, alternative oder zusätzliche Funktion ist, dass das Blütenöl von den Bienen in den Brutzellen verstrichen wird und der Verfestigung und Imprägnierung der Wände dient. Die wasserabweisenden Eigenschaften des Öls bieten einen wirkungsvollen Schutz vor Nässe. Zudem verharzt es durch Oxidation und bildet eine, einem Kunstharz ähnliche, impermeable Schicht. Auch wirkt es wahrscheinlich Infektionen durch Pilze und Bakterien entgegen (CANE et al. 1983, BuCHMAnN 1987, Schäffler \& Dötterl 2011, Kuhlmann 2014).

\section{Zusammensetzung des Blütenöls}

Nach der Entdeckung der Blütenöle war STEFAN Vogel auch der Erste, der sich mit der Chemie dieser Substanzen beschäftigte. Dabei setzte er neben chemischen Analysen auch vielfältige physikalisch-chemische und optische Methoden ein. Er führte umfangreiche chromatografische Trennungen (Dünnschichtchromatografie) der aus den Blüten gesammelten Rohsekrete durch, wobei die 
Methodik für die Blütenöle aber erst von ihm entwickelt werden musste. Für ihre weitere Analytik hätte er größere Mengen von Blütenölen benötigt, die aber aus den ihm zur Verfügung stehenden Blüten nicht extrahiert werden konnten. Selbst 30 Jahre später, also nach der Jahrtausendwende, scheiterten weitere Wissenschaftler an der Analytik, denn es stand einfach bei vielen Pflanzenarten nicht genug Rohsekret zu Verfügung. Eine ansehnliche Ölmenge lässt sich vor allem bei den Malpighiaceen gewinnen: eine Blüte von Stigmaphyllon ciliatum kann ca. $0,85 \mu \mathrm{l}$ Öl produzieren. Bei den winzigen Blüten aus der $O r$ nithocephalus-Verwandtschaft der Orchideen steht geschätzt nur ein Hundertstel davon zu Verfügung.

Die Vorgehensweise bei der Untersuchung der Blütenöle ist Folgende: nach wie vor wird das Öl mechanisch und von Hand gesammelt. Je nach Drüsentyp wird das Öl mit Kapillaren oder saugfähigem Filterpapier aufgenommen und in ein Lösungsmittel überführt. Üblicherweise wird heutzutage eine Mischung von tertiärem Butylmethylether (TBME) plus Methanol (2:1) verwendet. Die Probe wird bis zur Analyse kühl aufbewahrt. Da das Blütenöl keine Reinsubstanz darstellt, sondern aus einem Gemisch verschiedener Substanzen besteht, muss es aufgetrennt werden, d. h. die einzelnen Komponenten voneinander separiert werden. Anschließend werden diese getrennt analysiert. Bei Gemischen von Duftstoffen (ätherischen Ölen) ist dieses Verfahren verhältnismäßig einfach, denn die Substanzen lassen sich gaschromatografisch gut trennen und können dann in einem Arbeitsgang direkt im Massenspektrometer identifiziert werden. Fette Öle lassen sich hingegen nicht durch Gaschromatografie trennen, da sie zu schwerflüchtig sind. Deshalb müssen sie derivatisiert werden, also zu chemisch analogen, flüchtigen Verbindungen umgewandelt werden. Diese werden dann nach Auftrennung analysiert. Das Problem dieser Methode besteht in unbeabsichtigten Artefakten, die bei der Umwandlung entstehen. Diese Artefakte müssen jeweils interpretiert und aus den Ergebnissen eliminiert werden. Hier ist die Expertise von erfahrenen Chemikern gefragt, Biologen scheitern meist an den methodischen Schwierigkeiten.

Fette Öle (hier einfach Öle genannt) sind Triglyceride, also Ester des dreiwertigen Alkohols Glycerin mit verschiedenen Fettsäuren. Dabei können verschieden lange Fettsäuren involviert sein, die üblichen Kettenlängen betragen 12 bis 22 Kohlenstoffatome. In der Biosynthese werden jeweils $\mathrm{C}_{2}$-Einheiten zusammengefügt. Dadurch entstehen meist geradzahlige Ketten, die zudem unverzweigt sind. Es sind sowohl gesättigte als auch ungesättigte und mehrfach ungesättigte Fettsäuren vertreten, wobei ungesättigte Fettsäuren flüssigere Öle ergeben.

Typ A - (3R)-3-Acyloxyfettsäuren

Typ A $A_{1}$ (3R)-3-Acetoxyfettsäuren mit gerader C-Anzahl (Angelonia pubescens, Plantaginaceae)

Typ A 2 (3R)-3-Acyloxyfettsäuren mit gerader C-Anzahl, 3-Acetoxy- oder 3-(3-Methylbutanoyl)oxygruppe (Nierembergia scoparia, Solanaceae)

Typ $\mathrm{A}_{3}$ (3R)-3-Acetoxyfettsäuren mit gerader und ungerader C-Anzahl (Krameria lanceolata, Krameriaceae)

Typ B - Acylglycerine mit (3R)-3-Acetoxyacylresten

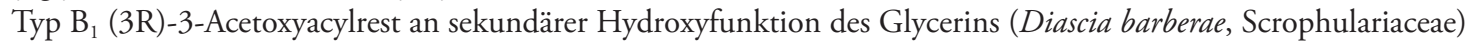

Typ $\mathrm{B}_{2}$ (3R)-3-Acetoxyacylrest an primärer Hydroxyfunktion des Glycerins (Lysimachia ciliata, Primulaceae)

Typ C - (3R)-3-Actoxyfettsäuren (Typ A1) und Acylglycerine (Typ B1) in ähnlichen Anteilen

(Trimezia martinicensis, Iridaceae)

Typ D - Mono- oder Diacylglycerine mit einem (nicht weiter funktionalisiertem) lang- und einem kurzkettigen Acylrest (Sisyrinchium micranthum, Iridaceae)

Typ E - Acetylierte Mono- und Dihydroxyfettsäuren (Malpighia coccigera, Malpighiaceae)

Typ F - Acylglycerine mit acetylierten Dihydroxyacylresten (Sigmatostalix huebneri, Orchidaceae)

Tab. 2: Chemische Vielfalt der Blütenöle (nach SEIPOLd 2004) 
Eine ganze Reihe verschiedenster Verbindungen wurden bisher im Öl der Ölblumen nachgewiesen. Dabei kommen auch Mono- und Diglyceride sowie freie Fettsäuren vor. Tabelle 2 gibt einen Überblick, der, ohne in die Details einzutreten, einen Eindruck der erstaunlichen chemischen Vielfalt vermitteln soll.

Wie dieser Überblick zeigt, ergibt sich eine verwirrende Vielfalt an involvierten Substanzen, die sich kaum adäquat in der Evolution der Pflanzenfamilien wiederspiegelt. Ein ambitioniertes deutsches Forschungsprojekt stellte sich kürzlich zur Aufgabe, eine große Zahl von Ölblumen bzw. deren Öle zu untersuchen. So wurden im Botanischen Garten München eine umfangreiche Sammlung von Ölblumen (mehr als 100 Arten) aufgebaut. Das Vorhaben scheiterte jedoch an der Komplexität der Blütenöle. So konnten in der vierjährigen Projektlaufzeit die Ölkomposition von nur etwa zehn verschiedenen Pflanzenarten, also lediglich einem Zehntel der in der Sammlung vertretenen Pflanzen, analysiert werden.

Wie die vielen nicht näher verwandten ölsammelnden Bienen eine Ölblume erkennen, ist bis heute nicht abschließend geklärt. Das Vorhandensein von Diacetin, eines flüchtigen acetylierten Glycerins, das bei zahlreichen Ölblumen nachgewiesen werden konnte, wäre eine plausible Erklärung (Schäffler et al. 2015). Die Diacetin-Synthese ist verlinkt mit der Biosynthese der Blütenöle, das Diacin könnte somit wegen seiner flüchtigen Eigenschaften Öl-Bienen als olfaktorisches Signal dienen.

\section{Die ölsammelnden Bienen}

Ähnlich wie sich Ölblumen in verschiedenen, nicht näher miteinander verwandten Angiospermenfamilien herausgebildet haben, haben sich auch verschiedene Gruppen von Bienen auf das Sammeln von Blütenölen spezialisiert. Nochmals ist festzuhalten, dass nur die Weibchen das Blütenöl sammeln, entweder in reiner Form (Öltracht) oder vermischt mit Pollen. Sowohl Männchen als auch die Weibchen nehmen Nektar an verschiedenen anderen Pflanzen zur

\section{Chemie der Blütenöle}

Fettsäure<smiles>CC(C)(C)CCCC(=O)O</smiles>

$\beta$-Hydroxyfettsäure

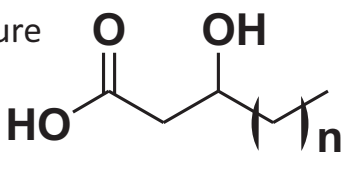

$\mathrm{n}=10,12,14$

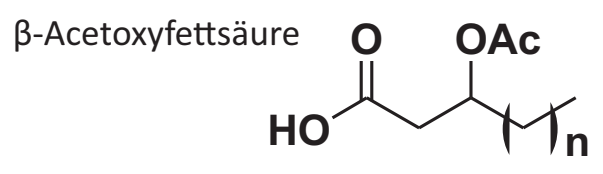

di-Acetoxyfettsäure

Acylglycerol

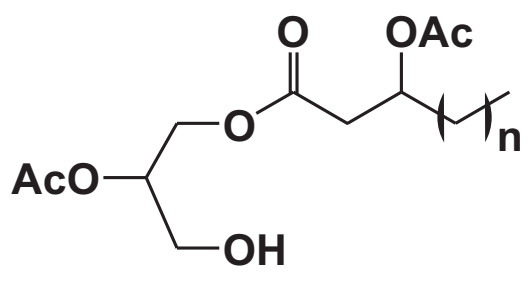

$\mathrm{n}=10,12,14$

Abb. 6: Strukturformeln von wichtigen Fettsäuren, die am Aufbau von Blütenölen beteiligt sind.

eigenen Energieversorgung mit der Zunge auf, während das Blütenöl immer mit den spezialisierten Beinen oder einer Bauchbürste (Ctenoplectra) gesammelt wird.

Bei den ölsammelnden Bienen handelt es sich um mehrere Untergruppen (Triben) der Echten Bienen (Apidae) sowie um zwei Untergruppen der Melittidae. Dass es sich um konvergent entstandene Entwicklungslinien handelt, erkennt man schon aus der Tatsache, dass die Methoden 
des Ölsammelns (mit zwei oder vier Beinen, mit Bauchbürste etc.) grundverschieden sind. Auch bewohnen die entsprechenden Bienenarten verschiedene Regionen der Erde.

Die Melittidae sind eine kleine Familie, die nach Michez et al. (2009) 16 Gattungen mit insgesamt etwa 200 Arten umfasst. Davon haben sich zwei Gattungen auf das Ölsammeln spezialisiert: Holarktisch verbreitete Schenkelbienen (Gattung Macropis) und die Gattung Rediviva mit Verbreitung im südlichen Afrika. Die Bienen dieser Gattungen sammeln das florale Öl auf verschiedene Weise. Die Arten von Macropis entnehmen das Öl mit vier Beinen, während die Rediviva-Arten sich mit vier Beinen an die
Blüten heften und mit den beiden, z. T. grotesk verlängerten Vorderbeinen das Öl aufnehmen. Näheres zu diesen Bienen und ihrer Biologie im nächsten Beitrag unserer Serie.

Die Apidae sind eine von sieben Familien der „Echten Bienen“ (Apidae). Ihre Klassifikation ist Gegenstand zahlreicher Publikationen und kontroverser Diskussionen. Molekulare Analysen und neue Methoden der phylogenetischen Rekonstruktion haben in den letzten zwanzig Jahren zu einem von traditionellen Vorstellungen stark abweichenden Verständnis ihrer Systematik und Evolution geführt (vgl. Danforth et al. 2013). Während Stefan Vogel die Anthophoridae und die Ctenoplectridae noch als eigene Familien betrachtete, werden

\begin{tabular}{|c|c|c|c|c|c|}
\hline Familie & $\begin{array}{l}\text { Unter- } \\
\text { familie }\end{array}$ & Tribus & $\begin{array}{l}\text { Gattungen } \\
\text { (Auswahl; Arten- } \\
\text { zahlen in Klammer) }\end{array}$ & Verbreitung & $\begin{array}{l}\text { Spezialisierung } \\
\text { auf bestimmte } \\
\text { Ölblumen }\end{array}$ \\
\hline \multirow[t]{4}{*}{ Apidae } & \multirow[t]{4}{*}{ Apinae } & Centridini & $\begin{array}{l}\text { Centris (ca. 230) } \\
\text { Epicharis }(35)\end{array}$ & Neotropis & $\begin{array}{l}\text { gering } \\
\text { hpts. } \\
\text { Malpighiaceae }\end{array}$ \\
\hline & & Tapinotaspidini & $\begin{array}{l}\text { Caenonomada (3) } \\
\text { Chalepogenus (21) } \\
\text { Paratetrapedia (32) } \\
\text { Tapinotaspis (4) } \\
\text { Tapinotaspoides (6) }\end{array}$ & $\begin{array}{l}\text { Neotropis } \\
\text { Neotropis } \\
\text { Neotropis } \\
\text { Neotropis } \\
\text { Neotropis }\end{array}$ & $\begin{array}{l}\text { gering } \\
\text { gering } \\
\text { gering } \\
\text { gering } \\
\text { gering }\end{array}$ \\
\hline & & Tetrapediini & Tetrapedia (ca. 13) & Neotropis & gering \\
\hline & & Ctenoplectrini & Ctenoplectra (16) & $\begin{array}{l}\text { W Afrika bis } \\
\text { SO Asien und } \\
\text { Australien }\end{array}$ & $\begin{array}{l}\text { Momordica, } \\
\text { Thladiantha } \\
\text { (Cucurbitaceae) }\end{array}$ \\
\hline \multirow[t]{2}{*}{ Melittidae } & \multirow[t]{2}{*}{ Melittinae } & Macropini & Macropis (16) & Holarktis & $\begin{array}{l}\text { Lysimachia } \\
\text { (Primulaceae) }\end{array}$ \\
\hline & & Melittini & Rediviva (26) & $\begin{array}{l}\text { Südliches } \\
\text { Afrika }\end{array}$ & $\begin{array}{l}\text { Diascia } \\
\text { (Scrophulariaceae) } \\
\text { Tritoniopsis } \\
\text { (Iridaceae) } \\
\text { Pterygodium, } \\
\text { Corycium, Disperis } \\
\text { (Orchidaceae) }\end{array}$ \\
\hline
\end{tabular}

Tab. 3: Stellung der ölsammelnde Bienen im System und allgemeine Informationen. Artenzahlen nach Revisionen und neueren Angaben von Roig Alsina (1999, 2003), Zanella (2002), Michez \& Patiny (2005), Schaefer \& Renner (2008), Melo \& Aguiar (2008), Aguiar \& Melo (2011), Kuhlmann \& Hollens (2014), und Martins et al. (2014). 
heute beide den Apidae eingegliedert, wobei die Gattungen der ersteren keine geschlossene Gruppe bilden, sondern über mehreren Untergruppen verteilt sind. Alle ölsammelnden Apiden gehören der Unterfamilie Apinae an, während die Unterfamilien Nomadinae und Xylocopinae keine solchen enthalten. Die wichtigsten Gattungen und ihre heutige Stellung sind in Tab. 3 zu finden.

Bei den Hauptgattungen der Apinae-Centridini, Centris und Epicharis, handelt es sich um große (Länge bis zu $3 \mathrm{~cm}$ ), am Thorax bräunlich- oder grau-pelzig behaarte Bienen, die trotz ihrer etwas plump wirkenden Gestalt schnelle und wendige Flieger sind. Die beiden Gattungen lassen sich dadurch auseinanderhalten, dass Epicharis lange, nach hinten gerichtete Borsten aufweist, die unmittelbar hinter den Augen ansetzen. Das Öl wird mit den Tarsen der Vorderbeine (bei einigen Arten zusätzlich der Mittelbeine) aufgenommen, die einen komplexen Bau aus Haaren, Borsten und spatelförmigen Elementen aufweisen (Abb. 8). Diese Strukturen kennt man an sich schon lange und hat sie ausgiebig zur Unterscheidung der Arten herangezogen. Doch blieb es Stefan Vogel und seiner akribischen Forschungsarbeit vorbehalten, den funktionellen Hintergrund aufzuklären, und

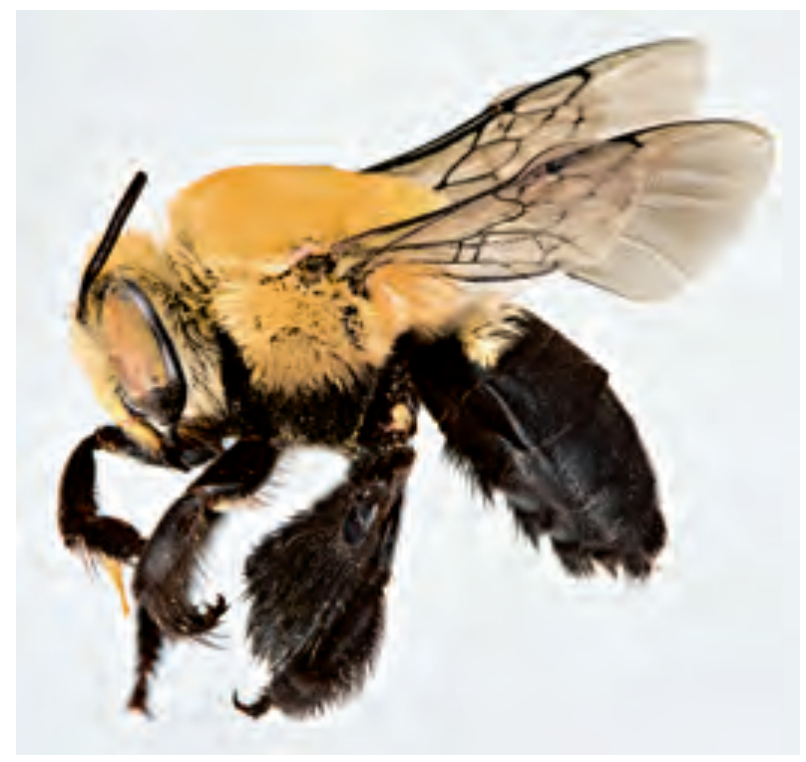

Abb. 7: Weibchen von Centris nitida (Apidae-Apinae-Centridini). Die Arten der großen Gattung Centris gehören zu den wichtigsten Bestäubern von neotropischen Ölblumen. (Foto: The Packer Lab, https://en.wikipedia.org/wiki/centris_nitida\#/media/file:centris_nitida_f.jpg). in großartigen Zeichnungen zu dokumentieren (vgl. Abb. 8).

Nach dem Sammelvorgang wird das Öl in einem kurzen Aufflug mittels der Mittelbeine in die voluminösen Bürsten (Scopae) der Hinterbeine verfrachtet. Diese Vorgangsweise ist jener der Honigbiene vergleichbar. Von dort wird das Öl im Nest herausgequetscht und, wie schon erwähnt, zur Imprägnierung der Nestwand bzw. mit Pollen vermischt als Larvenproviant verwendet.

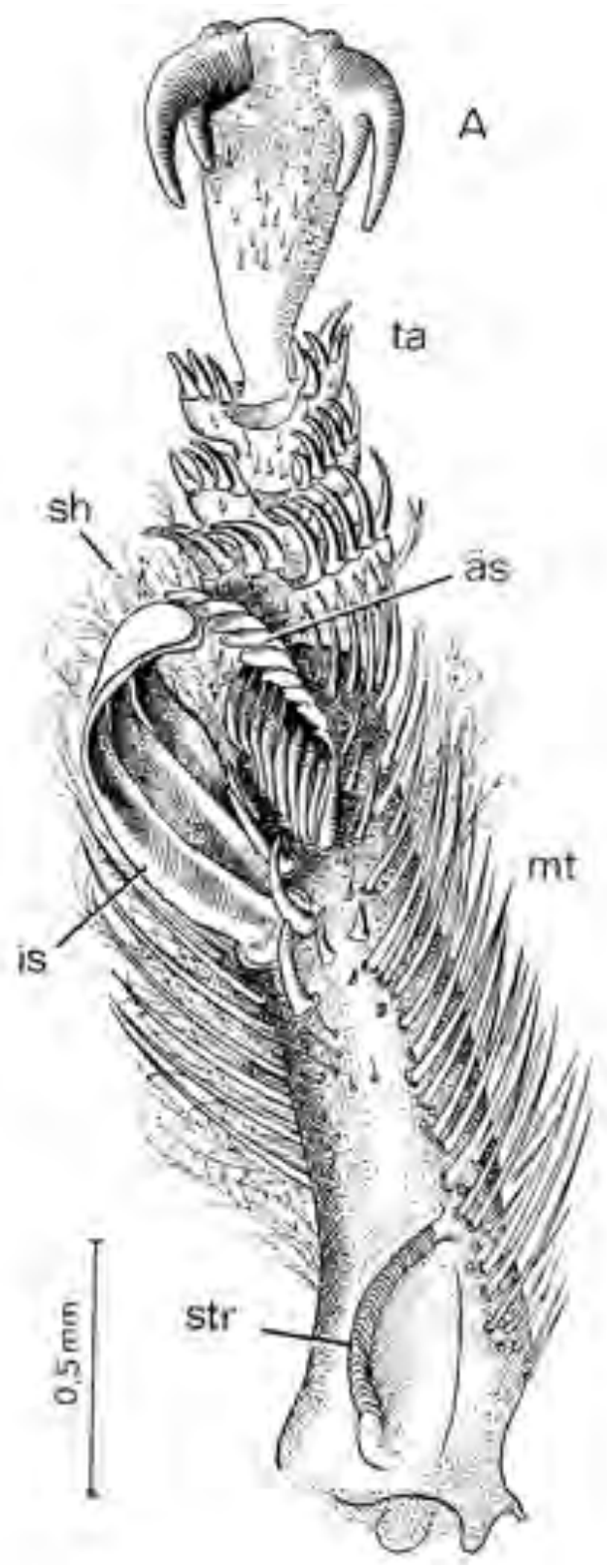

Abb. 8: Ölsammelapparat von Centris autrani, Ventralseite des rechten Vorderbeinendes; t Tarsus, mt Metatarsus, is innere Spatelreihe, äs äußere Spatelreihe, sh Saughaare, str Putzscharte (aus Vogel 1974: Abb. 50). 

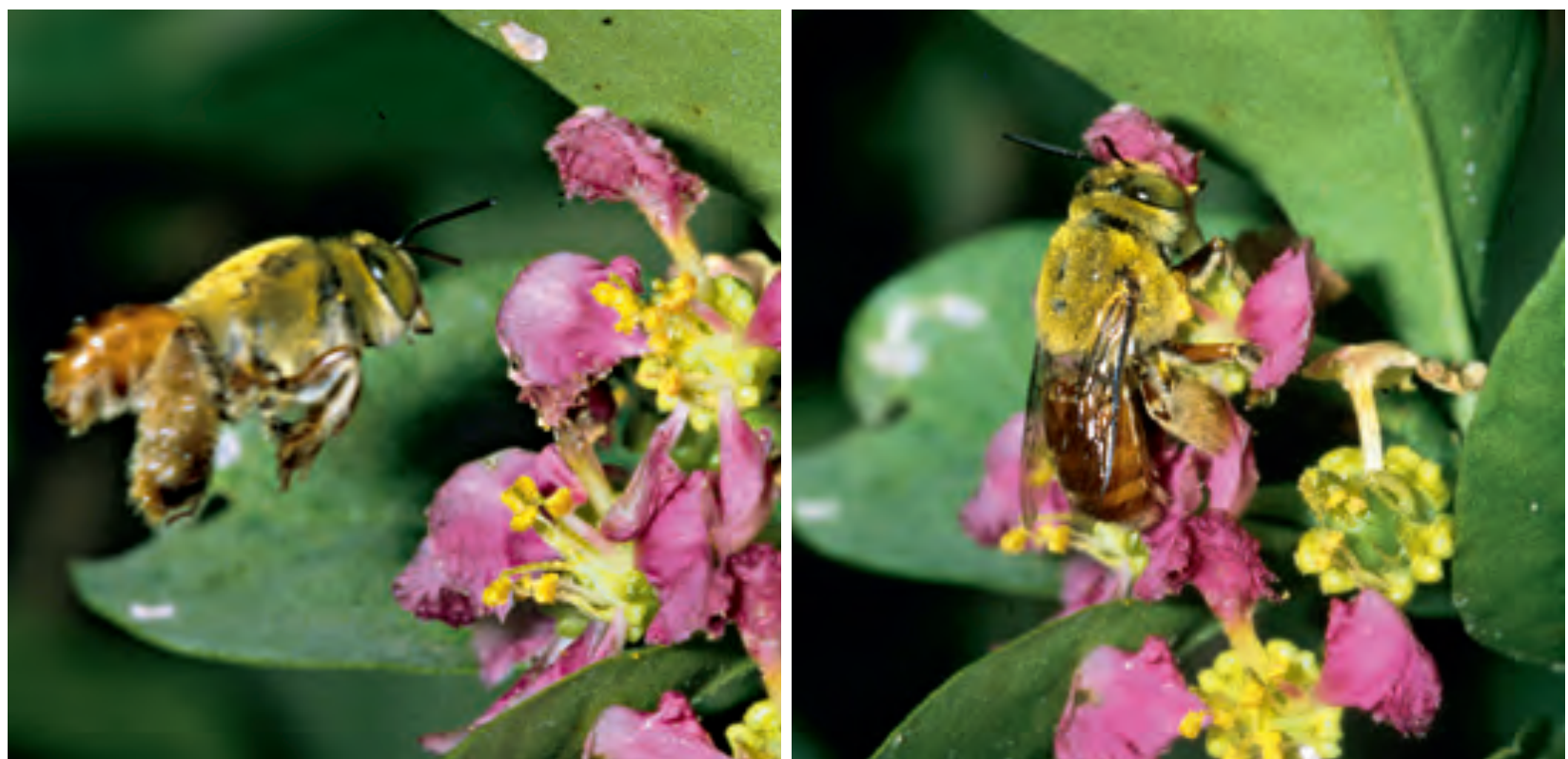

Abb. 9: Eine ölsammelnde Biene (Centris sp.) im Anflug und nach der Landung auf der Blüte von Malpighia emarginata (Malpighiaceae). Venezuela, Dept. Sucre, bei Cumana. (Fotos: G. Gerlach)

Ebenfalls ganz wichtige Ölsammler und Bestäuber in den Neotropen sind die (wenigen) Arten von Tapinotaspis und Tapinotaspoides. Diese Bienen sind wesentlich kleiner als die Centridinen. Durch ihre geringe Größe und schwarze Färbung ähneln sie in gewisser Weise den stachellosen Bienen (Meliponinen), können aber stechen. Auch bei Caenonomada, Chalepogenus, Paratetrapedia und Tetrapedia handelt es sich um relativ kleine Bienen, wobei erstere erst vor wenigen Jahren als Ölsammlerin erkannt wurde (Aguiar \& Melo 2009) und durch ihre wespenartige, schwarz-gelbe

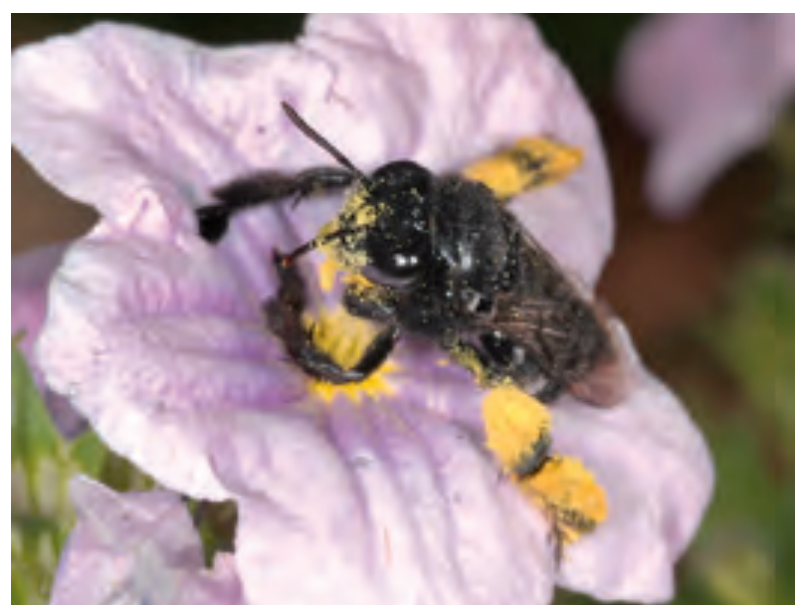

Abb. 10: Weibchen von Tapinotaspis chalybaea (ApidaeApinae-Taspinotaspidini) beim Ölsammeln auf einer Blüte von Nierembergia lineariifolia (Solanaceae). Die Scopae der Hinterbeine sind bereits mit Pollen und z. T. mit Öl gefüllt. Argentinien, Córdoba, Capilla del Monte. (Foto: A. A. CocuccI)
Warnfärbung gut zu erkennen ist. Auf diese Bienen und ihre mannigfaltigen Sammelvorrichtungen näher einzugehen würde den gegebenen Rahmen bei Weitem sprengen.

Die Spezialisierung auf bestimmte Ölblumen ist bei den erwähnten neotropischen Arten gering. Eher ausschlaggebend ist die Blütengröße. Große, offene Blüten werden vorwiegend von Centridinen besucht, während kleine und verwinkelt gebaute Blüten vorwiegend von Tapinotaspis und ähnlichen Bienen besucht werden. So sind etwa

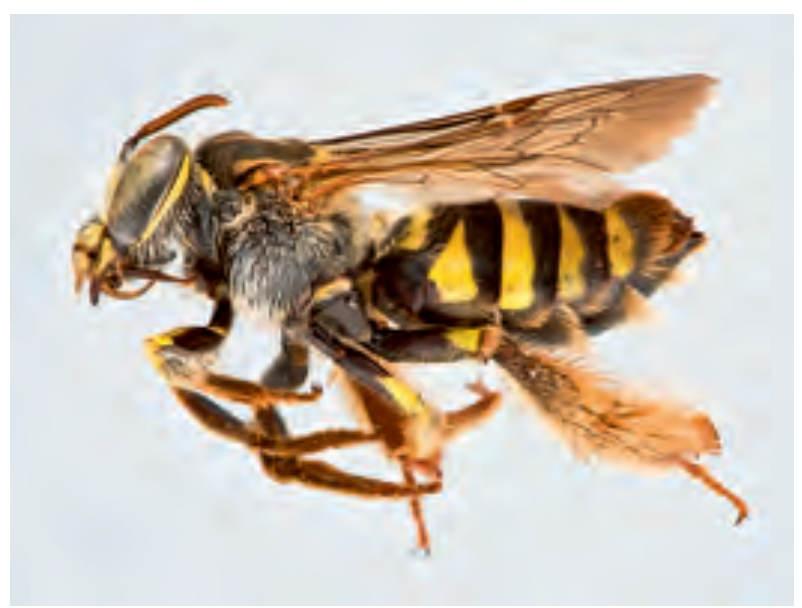

Abb. 11: Weibchen von Caenonomada unicalcarata (Apidae-Apinae-Tapinotaspidini). Diese kleinen ölsammelnden Bienen sehen sind durch die schwarz-gelbe Warnfärbung Wespen ähnlich. (Foto: The Packer Lab; https://commons. wikimedia.org/wiku/File:Caenonomada_unicalcarate_f.jpg) 
die großen Blüten von Calceolaria schickendantziana an die Bestäubung von Centris (C. autrani) angepasst, während die große Mehrzahl der Calceolarien (mit kleinen bis mittelgroßen Blüten) von Tapinotaspis-Arten bestäubt wird (Details bei Vogel 1974 und SÉrsic 2004). Daneben spielen aber auch strukturelle Merkmale sowohl der Blüten wie der Bienen eine Rolle.

Auf jene Bienen, die sich auf ganz bestimmte Blüten spezialisiert haben (und umgekehrt), wie beispielsweise Macropis auf Lysimachia oder Ctenoplectra auf Momordica und Thladiantha, wird im nächsten Artikel dieser Serie eingegangen.

\section{Zitierte und weiterführende Literatur}

Aguiar, A. J. C. \& Melo, G. A. R. 2009: Notes on oil sources for the bee genus Caenonomada (Hymenoptera, Apidae, Tapinotaspidini). - Revista Brasil. Entomol. 53: 154-156.

Aguiar, A. J. C. \& Melo, G. A. R. 2011: Revision and phylogeny of the bee genus Paratetrapedia Moure, with description of a new genus from the Andean Cordillera (Hymenoptera, Apidae, Tapinotaspidini). - Zool. J. Linn. Soc. 162: 351-442.

Buchmann, S. L. 1987: The ecology of oil flowers and their bees. - Ann. Rev. Ecol. Syst. 18: 343-369.

Buchmann, S. L. \& Buchmann, M. D. 1981: Anthecology of Mouriri myrtilloides (Melastomataceae, Memecylae), an oil flower in Panama. - Biotropica 13: 7-24.

Cane, J. H., Eickwort, G. C., Wesley, F. R. \& Spielholz, J. 1983: Foraging, grooming, and mating behaviors of Macropis nuda (Hymenoptera: Melittidae) and use of Lysimachia ciliata (Primulaceae) oils in larval provisions and cell lining. - Amer. Midl. Nat. 110: 257-264.

Cocucci, A. A. 1991: Pollination biology of Nierembergia (Solanaceae). - Plant Syst. Evol. 174: 17-35.|

Danforth, B. N., Cardinal, S., Praz, C., Almeida, E. A. B. \& Michez, D. 2013: The impact of molecular data on our understanding of bee phylogeny and evolution. - Ann. Rev. Entomol. 58: 57-78.

Goldblatt, P., Manning, J. C. \& Rudall, P. 1998: Iridaceae. - In: Kubitzki, K. (ed.): The families and genera of vascular plants. III. Monocotyledons, Lilianae (except Orchidaceae). - Berlin.

Houston, T. P., Lamont, B. B., Radford, S. \& ErringTON, S. G. 1993: Apparent mutualism between Verticordia nitens and $V$. aurea (Myrtaceae) and their oil-ingesting bee pollinators (Hymenoptera: Colletidae). - Austral. J. Bot. 41: 369-380.
Kiew, R., Weber, A. \& Burtt, B. L. 1998: Three new genera of Gesneriaceae from limestone of Peninsular Malaysia. - Beitr. Biol. Pflanzen 70: 383-403.

Kuhlmann, M. 2014: Nest architecture and use of floral oil in the oil-collecting South African solitary bee Rediviva intermixta (Cockerell) (Hymenoptera: Apoidea: Melittidae). - J. Nat. Hist. 48: 2633-2644.

Kuhlmann, M. \& Hollens, H., 2014: Morphology of oil-collecting pilosity of female Rediviva bees (Hymenoptera: Apoidea: Melittidae) reflects host plant use. - J. Nat. Hist. 49: 561-573.

Manning, J. \& Goldblatt, P. 2002: The pollination of Tritoniopsis parviflora (Iridaceae) by the oil-collecting bee Rediviva gigas (Hymenoptera: Melittidae): the first record of oil-secretion in African Iridaceae. - South African J. Bot. 68: 171-176.

Martins, A. C., Melo, G. A. R. \& Renner, S. S., 2014: The corbiculate bees arose from New World oil-collecting bees: Implications for the origin of pollen baskets. - Mol. Phylogenet. Evol. 80: 88-94.

Melo, G. A. R. \& Aguiar, A. J. C. 2008: New species of Tapinotaspoides (Hymenoptera, Apidae, Tapinotaspidini). Zootaxa 1749: 53-61.

Michez, D. \& Patiny, S. 2005: World revision of the oil-collecting bee genus Macropis Panzer 1809 (Hymenoptera: Apoidea: Melittidae) with a description of a new species from Laos. - Ann. Soc. Entomol. Fr. 41: 15-28.

Michez, D., Patiny, S. \& Danforth, B. N. 2009: Phylogeny of the bee family Melittidae (Hymenoptera: Anthophila) based on combined molecular and morphological data. - Syst. Entomol. 34: 574-597.

Possobom, C. C. F. \& Machado, S. R. 2017: Elaiophores: Their taxonomic distribution, morphology and functions. Acta Bot. Brasil. 31: 503-524.

Rasmussen, C. \& Olesen, J. M. 2000: Oil flowers and oil-collecting bees. - Norske Videnskap.-Akad., I. Mat. Naturvidenskap. Kl., Skrift., Ny Serie 39: 23-31.

Renner, S. S. \& Schaefer, H. 2010. The evolution and loss of oil-offering flowers - new insights from dated phylogenies for angiosperms and bees. - Phil. Trans. Roy. Soc. London, B, 365: 423-435.

Roig Alsina, A., 1999: Revision of the oil-collecting bees of the genus Chalepogenus Holmberg (Hymenoptera, Apidae, Tapinotaspidini). - Rev. Mus. Argentino Cienc. Nat., Nueva Serie 1: 67-101.

Roig Alsina, A: 2003: A revision of the bee genus Tapinotaspis Holmberg (Hymenoptera: Apidae, Tapinotaspidini). Mitt. Münch. Entomol. Ges. 93:45-53.

Schaefer, H. \& Renner, S. S. 2008: A phylogeny of the oil bee tribe Ctenoplectrini (Hymenoptera: Anthophila) based on mitochondrial and nuclear data: evidence for Early Eocene divergence and repeated out-of-Africa dispersal. - Mol. Phylogenet. Evol. 47: 799-811. 
Schaefer, H., Bartholomew, B. \& Boufford, D. E. 2012: Indofevillea jiroi (Cucurbitaceae), a new floral oil producing species from northeastern Myanmar. - Harvard Papers Bot. 17: 323-332.

Schäffler, I. \& Dötterl, S. 2011: A day in the life of an oil bee: Phenology, nesting $\&$ foraging behaviour. - Apidologie 42: 409-424.

Schäffler, I., Steiner, K. E., Haid, M., van Berkel, S. S., Gerlach, G., Johnson, S. D., Wessjohann, L. \& Dötterl, S. (2015): Diacetin, a reliable cue and private communication channel in a specialized pollination system. - Scientific Reports 5: 1-11; Art. no. 12779.

SEIPold, L. 2004: Blütenöle - Chemische Analyse, Biosynthese und Betrachtungen zur Entstehung von Ölblumen. - Diss. Univ. Halle-Wittenberg.

SÉrsic, A. N. 2004: Pollination in the genus Calceolaria. - Stapfia 82[fett]: 1-121.

Simpson, B. B. \& NefF, J. L. 1981: Floral rewards: alternatives to pollen and nectar. - Ann. Missouri Bot. Gard. 68: 301-322.

Steiner, K. E. 1985: The role of nectar and oil in the pollination of Drymonia serrulata (Gesneriaceae) by Epicharis bees (Anthophoridae) in Panama. - Biotropica 17: 217-229.

Steiner, K. E. \& Whitehead, V. B. 1991: Oil flowers and oil bees: further evidence for pollinator adaptation. - Evolution 45: 1493-1501.

Tate, J. A., Acosta, M. C., McDill, J., Moscone, E. A., Simpson, B. B. \& Cocucci, A. A. 2009: Phylogeny and character evolution in Nierembergia (Solanaceae): Molecular, morphological, and cytogenetic evidence. - Syst. Bot. 34: 198-206.

Troll, W. 1963: Kommission für Biologische Forschung. Bericht. I. Botanischer Teil. - Jahrb. Akad. Wiss. Lit. Mainz 1963: 115-134.

Troll, W. 1965: Kommission für Biologische Forschung. Bericht. I. Botanischer Teil. - Jahrb. Akad. Wiss. Lit. Mainz 1965: 110-131.

Vogel, S. 1969: Flowers offering fatty oil instead of nectar. XI International Botanical Congress (IBC), 24 Aug.-2 Sept. 1969, Seattle (US); Abstracts, p. 229.

Vogel, S. 1971: Ölproduzierende Blumen, die durch ölsammelnde Bienen bestäubt werden. - Naturwiss. 58: 58-59.

Vogel, S. 1973: Öl statt Nektar: Die „Ölblume“. - Umschau Wiss. Technik 73: 701-702.

Vogel, S. 1974: Ölblumen und ölsammelnde Bienen. - Abh. Akad. Wiss. Lit. Mainz, Math.-Naturwiss. Kl., Reihe Tropische und subtropische Pflanzenwelt 7: 285-547. Wiesbaden: Steiner.

Vogel, S. 1976a: Lysimachia: Ölblumen der Holarktis. Naturwiss. 63: 44.

Vogel, S. 1976b: Oil-collecting bees of the Old World and their flowers. - XV Int. Congr. Entomology (ICE), Washington D.C. (US), 19-27 Aug. 1976.
Vogel, S. 1981: Abdominal oil-mopping: a new type of foraging in bees. - Naturwiss. 67: 627-628.

Vogel, S. 1984: The Diascia flower and its bee: an oil-based symbiosis in Southern Africa. - Acta Bot. Neerl. 33(4): 509-518.

Vogel, S. 1986: Ölblumen und ölsammelnde Bienen. Zweite Folge: Lysimachia und Macropis. - Abh. Akad. Wiss. Lit. Mainz, Math.-Naturwiss. Kl., Reihe Tropische und subtropische Pflanzenwelt 54: 149-312. Wiesbaden.

Vogel, S. 1989: Fettes Öl als Lockmittel. Erforschung der ölbietenden Blumen und ihrer Bestäuber. - Jubiläumsband 1989 der Akad. Wiss. Lit. Mainz 1949-1989: 113-130.

Vogel, S. 1990a: Ölblumen und ölsammelnde Bienen. Dritte Folge. Momordica, Thladiantha und die Ctenoplectridae. Abh. Akad. Wiss. Lit. Mainz, Math.-Naturwiss. Kl., Reihe Tropische und subtropische Pflanzenwelt 73: 1-186. Wiesbaden.

Vogel, S. 1990b: History of the Malpighiaceae in the light of pollination ecology. - Mem. New York Bot. Gard. 55: 130-142.

Weber, A. \& Gerlach, G. 2018: Die großen wissenschaftlichen Leistungen von Stefan Vogel. Teil 4. Die „Parfümblumen“ und ihre prächtigen Bestäuber. - Palmengarten $\mathbf{8 1}$ : 133-148.

Whitehead, V. B. \& Steiner, K. E. 1992: Two new species of oil-collecting bees of the genus Rediviva from the summer rainfall region of South Africa (Hymenoptera, Apoidea, Melittidae). - Ann. South African Mus. 102: 4143-4164.

Zanella, F. C., 2002: Systematics and biogeography of the bee genus Caenonomada Ashmead, 1899 (Hymenoptera: Apidae: Tapinotaspidini). - Stud. Neotrop. Fauna Environm. 37: 249-261.

\section{Anschriften der Autoren}

Prof. Dr. Stefan Dötterl, AG Ökologie, Biodiversität und Evolution der Pflanzen, Universität Salzburg, Hellbrunner Straße 34, A-5020 Salzburg,

E-Mail: Stefan.Doetterl@sbg.ac.at

Dr. Günter Gerlach, Botanischer Garten München-Nymphenburg, Staatliche Naturwissenschaftliche Sammlungen Bayerns (SNSB), Menzinger Str. 61, D-80638 München, E-Mail: gerlach@extern.lrz-muenchen.de

Prof. i.R. Dr. Anton Weber, Department für Botanik und Biodiversitätsforschung, Universität Wien, Rennweg 14, A-1030 Wien, E-Mail: anton.weber@univie.ac.at 